\title{
Within- and between-series contrast in vowel identification: Full-vowel versus single-formant anchors
}

\author{
ROBERT ALLEN FOX \\ Ohio State University, Columbus, Ohio
}

\begin{abstract}
Subjects identified vowels from a [I]-[ع] continuum in both an equiprobable control condition and an anchored condition in which one stimulus (the anchor) occurred four times as often as any other stimulus. There were seven different anchors: (1) a full-vowel anchor representing the [1] endpoint of the test continuum; (2) a single-formant version of (1); (3) a full-vowel anchor representing the [I] endpoint but with a higher F0; (4) a single-formant version of (3); (5) a fullvowel anchor representing an [I] token but with a higher formant pattern than (3); (6) a singleformant version of (5); and (7) a pure tone anchor. The results demonstrated that the full-vowel anchors were more effective than either the single-formant anchors or the tone anchor in shifting the subjects' identification of the test tokens. Of the single-formant anchors, only (2) produced vowel contrast. The pattern of results suggests that only a portion of the vowel-contrast effect can be explained on the basis of processes operating at the auditory level.
\end{abstract}

The important of contextual contrast in speech perception, particularly in vowel identification, has been recognized at least since the work of Fry, Abramson, Eimas, and Liberman (1962) and Ladefoged and Broadbent (1957), but in the last decade it has been the subject of much renewed interest (e.g., Thompson \& Hollien, 1970; Morse, Kass, \& Turkienicz, 1976; Diehl, Elman, \& McCusker, 1978; Repp, Healy, \& Crowder, 1979; Sawusch \& Nusbaum, 1979; Sawusch, Nusbaum, \& Schwab, 1980; Crowder, 1981, 1982; Crowder \& Repp, 1984; Fox, 1985). Researchers have investigated this contextual effect using a variety of experimental procedures, including pairwise stimulus presentation (Repp et al., 1979), selective adaptation (Morse et al., 1976), and anchoring (Sawusch \& Nusbaum, 1979), but all have obtained a similar pattern of results, namely, that vowels are identified in contrast to the surrounding phonetic context. For example, a vowel token that is relatively ambiguous between the qualities [i] and [I] will more likely be identified as [i] when preceded by a prototypical [I] token and as [I] when preceded by a prototypical [i] token. Given such results, at least three different explanations for the vowel contrast effect have been suggested: (1) feature detector fatigue, (2) response bias, and (3) changes in auditory ground.

The feature-detector-fatigue hypothesis was developed primarily in conjunction with the research done on selective adaptation in consonant perception. It explains contextual effects in terms of the selective fatiguing of tuned phonetic (or auditory) detectors. In general, it has been

The author's mailing address is: Speech and Hearing Science Section, Department of Communication, Ohio State University, 324 Derby Hall, 154 N. Oval Mall, Columbus, OH 43210. dismissed as a viable explanation for vowel-contrast effects because there can often be a substantial amount of time between the context and the target (see discussion in Sawusch \& Nusbaum, 1979).

The response-bias hypothesis (e.g., Parducci, 1975), on the other hand, suggests that vowel-contrast effects (particularly as found in adaptation/anchoring studies) are a product of changes in subjects' labeling strategies, rather than of sensory fatigue or modification of internal perceptual referents. However, because the degree of vowel contrast obtained depends, at least in part, on the degree of spectral overlap between the context and the test series stimuli (Sawusch, 1977), and because such contrast effects occur even when subjects have been specifically alerted to the nature of the stimuli (see Sawusch \& Nusbaum, 1979), the weight of the evidence seems to rule out response bias as the only (or major) determinant of the vowel-contrast effect (see also Sawusch et al., 1980).

The third hypothesis suggests that contrast occurs when an auditory ground is established against which subsequent test stimuli are compared. This hypothesis seems to account for the preponderance of the available vowelcontrast data. However, the theory makes few or no assumptions about the actual perceptual mechanisms producing the contrast effects. Indeed, there may be several different mechanisms responsible. For example, Sawusch and Nusbaum (1983) suggest that contrast effects (at least for consonants) can be explained only with reference to at least two distinct levels of processing: auditory and phonetic. Furthermore, the work of Sawusch (1977) suggests that there are at least two different levels of auditory processing alone involved in the selective adaptation of consonants: a spectrally specific level of auditory analysis, possibly peripheral in nature, and an integrative level of auditory processing that is central. 
Recently, Crowder (1981, 1982; Crowder \& Repp, 1984) has developed a process-oriented model of vowel contrast hypothesizing a contrast mechanism which occurs at the auditory level of processing. In this model, vowels are represented in auditory memory in a form similar to a smudged (wide bandwidth) spectrogram. When two tokens are near one another in auditory memory (i.e., occur close in time), they show frequency-specific mutual lateral inhibition. That is, when the formant frequencies of two temporally adjacent vowels partially overlap, the energy in the shared frequencies is weakened, and, by contrast, the uninhibited frequencies within the formants are strengthened. This, in turn, may cause vowel quality shifts, since the perceived frequency peak of the formants may be higher or lower than the actual peak.

Crowder \& Repp (1984) found that vowel contrasts could be obtained with vowel tokens which had had both formant 2 and formant 3 removed. In fact, they found that these contrast effects were as powerful as those obtained using full three-formant vowels (in Experiment 1, using a [i]-[I] test continuum). Such results clearly imply the use of auditory (sensory) processing, and thus provide support for Crowder's theory. In particular, subjects did not identify the single-formant vowels as having the same phonetic quality as their full-formant counterparts; thus, response bias or other judgmental factors would seem to be eliminated.

However, Fox (1985) found that when vowels from different vocal-tract/source continua (i.e., vowel continua similar in phonetic quality but different in terms of fundamental frequency or absolute formant frequencies) were used in an anchoring experiment, cross-series anchors sometimes resulted in different degrees of contrast than those obtained with within-series anchors. Some contrasts seemed to be based upon normalized representations of the vowels, rather than upon their auditory images. Vowel contrast thus seems to result from mechanisms occurring at several levels of perceptual processing, rather than auditory processing alone.

The aim of this experiment was to obtain cross-series contrast data using single-formant anchors, and to compare these data with those obtained by Fox (1985) and Crowder and Repp (1984). If the cross-series anchoring effects are sensory in nature (i.e., stemming from lateral inhibition in auditory memory), as proposed by Crowder and Repp, then we would expect to find that singleformant anchors will be as effective as full-vowel anchors in shifting subjects' identification of ambiguous vowel stimuli. If other contrast mechanisms are responsible, then a different pattern of contrast effects should emerge.

\section{METHOD}

\section{Subjects}

The subjects were 56 undergraduates at Ohio State University who were native speakers of English with no known hearing impairment. Their participation fulfilled a course requirement.

\section{Stimuli}

A 7-step [hid]-[hed] vowel continuum was constructed using a cascade/parallel software synthesis program (Klatt, 1980). The
Table 1

First, Second, and Third Formant Frequencies for the Test Vowel Continuum

\begin{tabular}{cccc}
\hline Stimulus & F1 & F2 & F3 \\
\hline 1 & 400 & 1800 & 2570 \\
2 & 422 & 1780 & 2558 \\
3 & 443 & 1760 & 2547 \\
4 & 465 & 1740 & 2535 \\
5 & 587 & 1720 & 2523 \\
6 & 508 & 1700 & 2511 \\
7 & 530 & 1680 & 2500 \\
\hline
\end{tabular}

vowels were constructed with five formants, using the cascade mode of the synthesizer. The formant frequency values for F1, F2, and F3 used in the synthesis of the test series are shown in Table 1. The frequencies of F4 and F5 for all tokens and anchors were fixed at 3300 and $3850 \mathrm{~Hz}$, respectively. All vowels were steady-state and each stimulus was $420 \mathrm{msec}$ in duration. The formant bandwidths for F1, F2, and F3 were 50,100 , and $140 \mathrm{~Hz}$, respectively, for the steady-state portions of the vowels. The bandwidths of F4 and F5 were fixed at 250 and $200 \mathrm{~Hz}$, respectively. Fundamental frequency (F0) for each token began at $130 \mathrm{~Hz}$ and fell linearly to $100 \mathrm{~Hz}$ after $360 \mathrm{msec}$ where it remained until the end of the token. The [h] of each token was produced by using an unvoiced, aperiodic hiss source with the given formant frequency values. The formant transitions to the final [d] were $30 \mathrm{msec}$ in duration and went from the formant values specified in Table 1 to 200,1600 , and $2600 \mathrm{~Hz}$ for F1, F2, and F3, respectively. Seven anchor stimuli were constructed. The A1 full-vowel anchor ${ }^{1}$ represented the [hid] end of the test continuum. The Al single-formant anchor was produced by utilizing the same synthesis parameter values as were used for the Al full-vowel anchor, except that the parallel synthesis mode was used with only the amplitude of $F 1$ above zero $(60 \mathrm{~dB})$.

Anchors Bl and Cl were both hid tokens from parallel [hid][hed] continua. The Bl anchors differed from their Al counterparts in terms of F0 only. In particular, the F0 of the B1 anchors began at $220 \mathrm{~Hz}$ and fell to $190 \mathrm{~Hz}$ after $360 \mathrm{msec}$. The $\mathrm{Cl}$ anchors differed from the A1 anchors in terms of both F0 and absolute formant frequencies, but still represented the formant patterns necessary to produce the appropriate /I/ phonetic quality for a prototypical hid token. The frequencies for F1, F2, and F3 for the steady-state portions of the C1 full-vowel anchor were 450,2300, and $3000 \mathrm{~Hz}$, respectively, with the same formant bandwidths as were used for the Al and Bl anchors and the test continuum. The formant transitions to the final [d] were $30 \mathrm{msec}$ in duration and went from their steady-state frequencies to 400,2000 , and $2900 \mathrm{~Hz}$ for $F 1, F 2$, and F3, respectively. The F0 contour was the same for both the $\mathrm{B} 1$ and $\mathrm{Cl}$ anchors. The $\mathrm{B} 1$ and $\mathrm{Cl}$ single-formant anchors were produced by utilizing the parallel synthesis mode; the synthesis parameter settings of their full-vowel counterparts were used, with only the amplitude of $F 1$ above zero. The amplitudes of the single-vowel anchors were matched with those of the fullvowel anchors.

The test-vowel continuum and the Al anchors represent the output of a long articulatory tract with a relatively low F0; the $\mathrm{Bl}$ anchors represent the output of a long articulatory tract with a relatively high F0; and the $\mathrm{Cl}$ anchors represent the output of a shorter articulatory tract with a relatively high $\mathrm{F} 0$. The seventh anchor was a $400 \mathrm{~Hz}$ tone that was approximately $420 \mathrm{msec}$ in duration. The frequency of this tone anchor was chosen to match the F1 frequency of the anchors and best represents a nonspeech analogue to the Al single-formant anchors. Inclusion of the tone anchor will allow us to discover if contrast can be obtained at the auditory level alone, that is, whether single-formant contrast can be produced without the involvement of a "speech mode" of perception. Data reproduced here using the A1, B1, and C1 full-vowel anchors were obtained as part of an earlier experiment (reported in Fox, 1985).

A baseline tape and seven anchor tapes were constructed. In the baseline tape each of the test tokens occurred 20 times in random 
order. The anchor tapes contained 20 presentations of the seven test tokens plus 60 presentations of one anchor in random order.

\section{Procedure}

Subjects were randomly divided into seven groups of 8 subjects each. Each subject heard first the baseline tape and then one of the seven anchor tapes. Subjects were required to listen to a token and then identify the token as either hid or head. In addition, each subject was required to rate each identification on a 4-point scale indicating the subject's confidence that the response was correct. These identification-plus-rating responses were converted into values on an 8-point scale with 1 representing a very confident hid response and 8 representing a very confident head response. Subjects were not required to identify the single-formant and tone anchors that occurred in the anchor condition (and which were clearly discernible from the test tokens), but were to respond simply by placing an " $X$ " in the appropriate spot on the response sheets.

\section{RESULTS AND DISCUSSION}

The results of this experiment are shown in Figure 1. Each of the plots in this figure shows the 8-point scaled identification functions collapsed across subjects in both the baseline and anchor conditions. Note (in the leftmost three panels) the significant shifts in the phoneme boundaries (the $50 \%$ crossover point calculated by linear interpolation) obtained using full-vowel anchors $\mathrm{A} 1$ [t $(7)=$ $2.80, \mathrm{p}<.03$, mean boundary shift $=-.58]$ and $\mathrm{Bl}$ $[\mathrm{t}(7)=11.4, \mathrm{p}<.001$, mean boundary shift $=-.94]$. Although the $\mathrm{C} 1$ full-vowel anchor produced a boundary shift in the same direction, it was not significant $[t(7)=$ $1.22, p=.25$, mean boundary shift $=-.25]$. However, of the single-formant anchors, only A1 produced an even marginally significant shift in the identification function $[\mathrm{t}(7)=2.31, \mathrm{p}=.054$; Wilcoxon signed ranks: $\mathrm{z}=1.96$, $\mathrm{p}<.05$, mean boundary shift $=-.25$ ). Unlike the B1
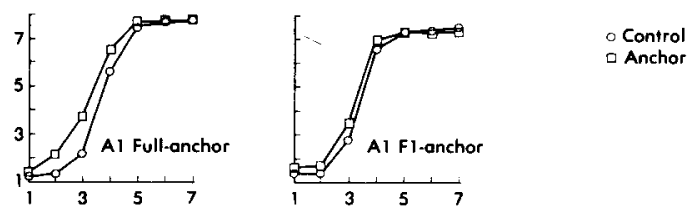

- Anchor
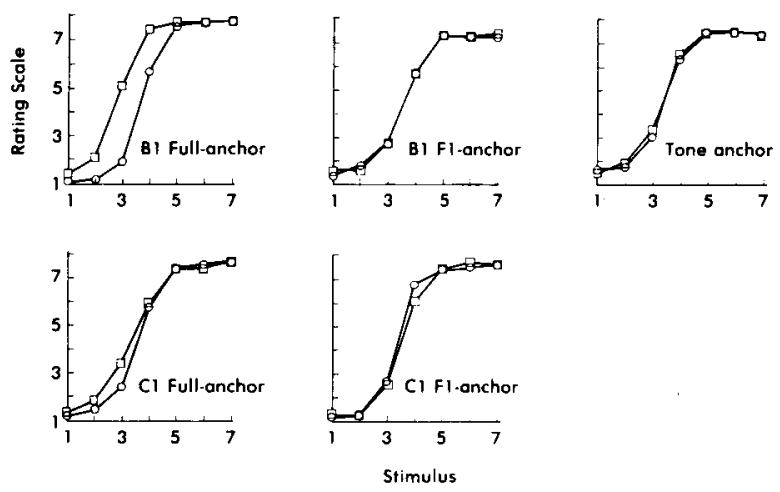

Figure 1. The effect of seven anchors on the identification of vowels along an $[\mathrm{I}]-[\varepsilon]$ continuum. The full-vowel anchors are plotted in the leftmost panels, the single-formant anchors in the middle panel, and the tone anchor in the rightmost panel. full-vowel anchor, which produced a significantly greater shift than did the Al full-vowel anchor, the B1 singleformant anchor did not produce a significant shift in the identification function $[\mathrm{t}(7)=1.07, \mathrm{p}>.58$, mean boundary shift $=.06]$. Neither the $\mathrm{Cl}$ single-formant anchor $[\mathrm{t}(7)=.58, \mathrm{p}>.58$, mean boundary shift $=.06]$ nor the tone anchor $[t(7)=1.82, p>.11$, mean boundary shift $=.025]$ produced a significant shift in the identification function. The boundary shifts produced by the six vowel anchors only were analyzed together, using a $3 \times 2$ analysis of variance with the factors anchor continuum (the vocal-tract/source variations) and anchor type (full vowel vs. single formant). The analysis revealed significant main effects due to anchor continuum $[F(2,42)$ $=4.98, \mathrm{p}<.02]$ and anchor type $[\mathrm{F}(1,42)=16.2, \mathrm{p}$ $<.001]$, but no significant interaction effect $[F(2,42)=$ $1.87, \mathrm{p}>.16]$.

These results seem to support at least three generalizations. First, it is not necessarily the case that singleformant contrast is as powerful as full-vowel contrast, even when no differences in F0 or overall formant patterns are involved. In particular, the boundary shift produced by the A1 single-formant anchor is less than onehalf of the boundary shift produced by the A1 full-vowel anchor. This difference is at least marginally significant $[\mathrm{t}(14)=2.12, \mathrm{p}=.052]$. Although Crowder and Repp (1984, Experiment 1) found no difference between fullvowel and single-formant contrast using a [i]-[I] test series, they did obtain a significant difference between fullvowel and single-formant contrast when using a $[\varepsilon]-[æ]$ test series (Experiment 2). Crowder and Repp suggested that the discrepancy in the amount of contrast produced by the full-vowel versus single-formant precursors stemmed from the fact that in Experiment 1, the frequency of only $\mathrm{F} 1$ changed along the vowel continuum (F2 and F3 were fixed), whereas in Experiment 2, the frequencies of both F1 and F2 changed (F3 was fixed). They suggested that an ambiguous item was receiving potential contrasting influences from both $F 1$ and $F 2$ in Experiment 2, but from only F1 in Experiment 1. For the data presented here, it can be argued that the ambiguous test items received contrasting influences from F1, F2, and F3 in the case of the full-vowel anchors, but from only $F 1$ in the case of the single-formant anchors. In general, then, these data suggest, perhaps not unexpectedly, that both formant 1 and formant 2 (the two most important determinants of vowel quality) contribute to the contrast effect to some (probably unequal) extent.

Second, these data give at least limited support for Crowder's notion of physical channel distinctions in auditory memory. In particular, Crowder $(1981,1982)$ suggested that the lateral inhibition responsible for the vowelcontrast effect occurs only between tokens that are similar or identical in physical channel. If we assume that fundamental frequency differences are sufficient to produce channel distinctions, then the results presented here for the single-formant anchors, although not for the full-vowel anchors, show the pattern one would expect from withinchannel mutual lateral inhibition. 
Finally, these data provide evidence that not all vowelcontrast effects arise from lateral inhibition in auditory memory. In particular, although the B1 full-vowel anchor produced a significant contrast effect, the B1 singleformant anchor did not. If we explain the Al singleformant contrast on the basis of within-channel frequencyspecific lateral inhibition, then we might suggest that the high-F0 B1 single-formant anchor fails to produce significant contrast because it occupies a different channel from the low-F0 test series. This could not, however, explain why the B1 full-vowel anchor produces significant contrast, since it should presumably occupy the same channel as does the B1 single-formant anchor. In addition, note that the B1 full-vowel anchor produced a boundary shift significantly greater than that produced by the Al full-vowel anchor $[t(14)=2.86, p<.02]$. This also is not readily explained in terms of formant-frequencyspecific inhibition, since the $\mathrm{A} 1$ and $\mathrm{B} 1$ anchors have the same formant structure and differ only in terms of fundamental frequency. Fox (1985) suggested that the differences in boundary shift produced by the A1 and B1 fullvowel anchors are due to the possibility that at least a portion of the contrast effect takes place following talker normalization (cf. Ladefoged \& Broadbent, 1957; Fant, 1966; Gerstman, 1968; Lieberman, Crelin, \& Klatt, 1972). For example, if listeners normalize (or rescale) the formant structure of a vowel to eliminate vocal-tract length differences between talkers, and utilize F0 (which is negatively correlated with vocal-tract length, at least across sexes) to estimate this length (and to determine the scaling factor), the perceived phonetic quality of a given formant pattern will covary with F0. The rescaled vowel in the B1 full-vowel anchor would then be perceived as having a lower F1 than the Al full-vowel anchor (see Slawson, 1968; Fujisaki \& Kawashima, 1968). This would result in the B1 full-vowel anchor's producing a greater phoneme-boundary shift than the A1 full-vowel anchor (see Fox, 1985, for a description of other relevant crossseries contrast effects and a related discussion of this view). We assume that this normalization process is phonetic in nature, takes place after nonspeech auditory processes, and does not directly involve lateral inhibition in auditory memory.

To conclude, these data are consistent with the suggestion by Sawusch et al (1980) that at least two different perceptual processes are involved in the production of vowel-contrast effects (although Sawusch et al.'s conception of these processes is slightly different from mine). The first process appears to be primarily sensory in nature and to operate at the auditory level, limited to contrast within a physical channel. The second process appears to operate at the phonetic level and to involve normalized vowel images (it is not clear whether or not this second level of contrast should be modeled in terms of mutual lateral inhibition). Future work in this area is needed to ascertain both the number and the nature of these different levels of vowel contrast.

\section{REFERENCES}

Crowder, R. G. (1981). The role of auditory memory in speech perception and discrimination. In T. Meyers, J. Laver, \& J. Anderson (Eds.), The cognitive representation of speech. Amsterdam: NorthHolland.

CrowDER, R. G. (1982). Decay of auditory memory in vowel discrimination. Journal of Experimental Psychology: Learning, Memory, \& cognition, 8, 153-162.

Crowder, R. G., \& RePP, B. H. (1984). Single formant contrast in vowel identification. Perception \& Psychophysics, 35, 372-378.

Diehl, R. L., Elman, J. L., \& McCusker, S. B. (1978). Contrast effects in stop consonant identification. Journal of Experimental Psychology: Human Perception \& Performance, 4, 599-609.

FANT, G. (1973). A note on vocal tract size factors and nonuniform F-pattern scalings. In G. Fant, Speech sounds and features. Cambridge, MA: MIT Press. (Reprinted from Speech Transmission Laboratory Quarterly Progress and Status Report, April 1966)

Fox, R. A. (1985). Auditory contrast and speaker quality variation in vowel perception. Journal of the Acoustical Society of America, 77 , 1552-1559.

Fru, D., Abramson, A., Eimas, P. D., \& Liberman, A. M. (1962). The identification and discrimination of synthetic vowels. Language \& Speech, 5, 171-189.

FUjISAKI, H., \& KaWASHIMA, T. (1968). The roles of pitch and higher formants in the perception of vowels. IEEE Transactions on Audio and Electroacoustics, $\mathrm{AU}-16,73-77$.

Gerstman, L. J. (1968). Classification of self-normalized vowels. IEEE Transactions of Audio and Electroacoustics, AU-16, 78-80.

KLatT, Dennis H. (1980). Software for a cascade/parallel formant synthesizer. Journal of the Acoustical Society of America, 67, 971-995.

LADEFOGED, P. \& BROADBENT, D. E. (1957). Information conveyed by vowels. Joumal of the Acoustical Society of America, 29, 98-104.

Lieberman, P., Crelin, E. S., \& Klatt, D. (1972). Phonetic ability and related anatomy of the newborn, adult human, Neanderthal man, and the chimpanzee. American Anthropologist, 74, 287-307.

Morse, P., Kass, J. E., \& Turkienicz, H. (1976). Selective adaptation of vowels. Perception \& Psychophysics, 19, 137-143.

Parducci, A. (1975). Contextual effects: A range-frequency analysis. In E. C. Carterette \& M. P. Friedman (Eds.), Handbook of Perception, Vol. II. New York: Academic Press.

RepP, B. H., Healy, A. F., \& Crowder, R. G. (1979), Categories and contexts in the perception of isolated steady-state vowels. Journal of Experimental Psychology: Human Perception \& Performance, $5,129-145$.

Sawusch, J. R. (1977). Peripheral and central processes in selective adaptation of place of articulation in stop consonants. Journal of the Acoustical Society of America, 62, 738-750.

Sawusch, J. R., \& Nusbaum, H. C. (1979). Contextual effects in vowel perception I: Anchor-induced contrast effects. Perception \& Psychophysics, 25, 292-302.

SawusCh, J. R., \& Nusbaum, H. C. (1983). Auditory and phonetic processes in place perception for stops. Perception \& Psychophysics, $34,560-568$.

Sawusch, J. R., Nusbaum, H. C., \& Schwab, E. C. (1980). Contextual effects in vowel perception II: Evidence for two processing mechanisms. Perception \& Psychophysics, 27, 421-434.

SLAwson, A. W. (1968). Vowel quality and musical timbre as functions of spectrum envelope and fundamental frequency. Journal of the Acoustical Society of America, 43, 87-101.

Thompson, C. L., \& Hollien, H. (1970). Some contextual effects on the perception of synthetic vowels. Language and Speech, 13, 1-13.

\section{NOTE}

1. The stimuli have been labeled using the scheme adopted by Fox (1985), so that comparisons between the two studies may be made more easily. 